Bull. Korean Math. Soc. 48 (2011), No. 1, pp. 17-21

DOI 10.4134/BKMS.2011.48.1.017

\title{
DUALITY OF CO-POISSON HOPF ALGEBRAS
}

\author{
Sei-Qwon Oh and Hyung-Min Park
}

\begin{abstract}
Let $A$ be a co-Poisson Hopf algebra with Poisson co-bracket $\delta$. Here it is shown that the Hopf dual $A^{\circ}$ is a Poisson Hopf algebra with Poisson bracket $\{f, g\}(x)=\langle\delta(x), f \otimes g\rangle$ for any $f, g \in A^{\circ}$ and $x \in A$ if $A$ is an almost normalizing extension over the ground field. Moreover we get, as a corollary, the fact that the Hopf dual of the universal enveloping algebra $U(\mathfrak{g})$ for a finite dimensional Lie bialgebra $\mathfrak{g}$ is a Poisson Hopf algebra.
\end{abstract}

Let $G$ be a Lie group with Lie algebra $\mathfrak{g}$. Then its coordinate ring $\mathcal{O}(G)$ is a Hopf algebra and can be replaced by the Hopf dual $U(\mathfrak{g})^{\circ}$ of the universal enveloping algebra $U(\mathfrak{g})$. In fact, it is well-known that $U(\mathfrak{g})^{\circ}$ is equal to $\mathcal{O}(G)$ if $G$ is connected and simply connected. Moreover it is convenient to work on $U(\mathfrak{g})^{\circ}$ instead of $\mathcal{O}(G)$ since $U(\mathfrak{g})^{\circ}$ has a natural grading. For instance, see [3, Chapter 2] and [2].

Recall that a Lie group $G$ is said to be a Poisson Lie group if its coordinate $\operatorname{ring} \mathcal{O}(G)$ is a Poisson Hopf algebra. If $G$ is a Poisson Lie group, then its Lie algebra $\mathfrak{g}$ becomes a finite dimensional Lie bialgebra with a co-bracket $\delta$ and the universal enveloping algebra $U(\mathfrak{g})$ is a co-Poisson Hopf algebra with Poisson co-bracket extended naturally from $\delta$ (See $[1, \S 6.2])$. Thus its Hopf dual $U(\mathfrak{g})^{\circ}$ would be a Poisson Hopf algebra with Poisson bracket induced by $\delta$. At this moment, we would show the fact $\{f, g\} \in U(\mathfrak{g})^{\circ}$ for all $f, g \in U(\mathfrak{g})^{\circ}$.

Let $A$ be a co-Poisson Hopf algebra. Since the concept of a co-Poisson Hopf algebra is a dual concept of Poisson Hopf algebra, the Hopf dual $A^{\circ}$ of $A$ is anticipated a Poisson Hopf algebra. Here we give a complete proof that the Hopf dual $A^{\circ}$ is a Poisson Hopf algebra in the case that $A$ is an almost normalizing extension over the ground field and we get, as a corollary, the fact that $U(\mathfrak{g})^{\circ}$ is a Poisson Hopf algebra if $\mathfrak{g}$ is a finite dimensional Lie bialgebra.

Assume throughout that $\mathbf{k}$ denotes a field of characteristic zero and all vector spaces are over $\mathbf{k}$.

Received April 13, 2009.

2010 Mathematics Subject Classification. 17B62, 17B63, 16W30.

Key words and phrases. co-Poisson Hopf algebra, Poisson Hopf algebra.

This work was supported by the Korea Research Foundation Grant funded by the Korean Government, KRF-2008-313-C00021. 
Recall the definition of co-Poisson Hopf algebra. Let $A=(A, \iota, \mu, \epsilon, \Delta, S)$ be a Hopf algebra over $\mathbf{k}$. Let $\tau$ be the flip on $A \otimes A$, that is, $\tau$ is a k-linear map defined by

$$
\tau: A \otimes A \longrightarrow A \otimes A, \quad x \otimes y \mapsto y \otimes x
$$

and set

$$
\tau_{12}=\tau \otimes 1, \quad \tau_{23}=1 \otimes \tau .
$$

A Hopf algebra $A$ is said to be a co-Poisson Hopf algebra if there exists a skewsymmetric k-linear map $\delta: A \longrightarrow A \otimes A$, called a Poisson co-bracket, satisfying the following conditions:

(i) (co-Jacobi identity)

$$
(\delta \otimes 1) \circ \delta+\tau_{12} \circ \tau_{23} \circ(\delta \otimes 1) \circ \delta+\tau_{23} \circ \tau_{12} \circ(\delta \otimes 1) \circ \delta=0 .
$$

(ii) (co-Leibniz rule)

$$
(\Delta \otimes \mathrm{id}) \circ \delta=(\mathrm{id} \otimes \delta) \circ \Delta+\tau_{23} \circ(\delta \otimes \mathrm{id}) \circ \Delta .
$$

(iii) $(\Delta$-derivation $)$

$$
\delta(a b)=\delta(a) \Delta(b)+\Delta(a) \delta(b)
$$

for all $a, b \in A$.

Definition 1 ([4, 1.6.10]). An algebra $R$ over $\mathbf{k}$ is said to be an almost normalizing extension over $\mathbf{k}$ if $R$ is a finitely generated $\mathbf{k}$-algebra with generators $x_{1}, \ldots, x_{n}$ satisfying the condition

$$
x_{i} x_{j}-x_{j} x_{i} \in \sum_{\ell=1}^{n} \mathbf{k} x_{\ell}+\mathbf{k}
$$

for all $i, j$.

Lemma 2. Let $R$ be an almost normalizing extension of $\mathbf{k}$ with generators $x_{1}, \ldots, x_{n}$. Then $R$ is spanned by all standard monomials

$$
x_{1}^{r_{1}} x_{2}^{r_{2}} \cdots x_{n}^{r_{n}}, \quad r_{i}=0,1, \ldots
$$

together with the unity 1.

Proof. This follows immediately from induction on the degree of monomials.

Note that the Hopf dual $A^{\circ}$ of a Hopf algebra $A$ consists of

$$
A^{\circ}=\left\{f \in A^{*} \mid f(I)=0 \text { for some cofinite ideal } I \text { of } A\right\},
$$

where $A^{*}$ is the dual vector space of $A$. 
Theorem 3. Let $A$ be a co-Poisson Hopf algebra with Poisson co-bracket $\delta$. If $A$ is an almost normalizing extension over $\mathbf{k}$, then the Hopf dual $A^{\circ}$ is a Poisson Hopf algebra with Poisson bracket

$$
\{f, g\}(x)=\langle\delta(x), f \otimes g\rangle, \quad x \in A
$$

for any $f, g \in A^{\circ}$, where $\langle\cdot, \cdot\rangle$ is the natural pairing between the vector space $A \otimes A$ and its dual vector space.

Proof. Step 1. The Poisson bracket (1) is well-defined. That is, $\{f, g\} \in A^{\circ}$ for every $f, g \in A^{\circ}$ : There exist cofinite ideals $I, J$ of $A$ such that $f(I)=$ 0 and $g(J)=0$. Since the canonical map $A /(I \cap J) \longrightarrow A / I \times A / J$ is a monomorphism, the ideal $I \cap J$ is also cofinite. Set

$$
K=(I \cap J) \otimes A+A \otimes(I \cap J) .
$$

Note that $\langle K, f \otimes g\rangle=0$. The canonical map from $[A /(I \cap J)] \otimes[A /(I \cap J)]$ into $(A \otimes A) / K$ is surjective and thus $(A \otimes A) / K$ is finite dimensional.

Note that $A$ is spanned by the standard monomials

$$
x_{1}^{r_{1}} x_{2}^{r_{2}} \cdots x_{n}^{r_{n}}, \quad r_{i}=0,1, \ldots
$$

together with the unity 1 by Lemma 2 . For each $i=1,2, \ldots, n$, the set of cosets

$$
\left\{\delta\left(x_{i}^{k}\right)+K \mid k=1,2, \ldots\right\}
$$

is linearly dependent since $(A \otimes A) / K$ is finite dimensional and thus there exists a nonzero polynomial $h \in \mathbf{k}[x]$ such that $\delta\left(h\left(x_{i}\right)\right) \in K$, where $x$ is an indeterminate. Consider the set

$$
S=\left\{0 \neq h \in \mathbf{k}[x] \mid \delta\left(h\left(x_{i}\right)\right) \in K\right\}
$$

Note that $S$ is an infinite set since $K$ is an ideal and $S$ is not empty. For instance, if $h \in S$, then $h^{k} \in S$ for all positive integer $k$ by the $\Delta$-derivation of $\delta$. Since $S$ is an infinite set and $(A \otimes A) / K$ is finite dimensional, there exists a nonzero polynomial $h_{i} \in S$ such that $\Delta\left(h_{i}\left(x_{i}\right)\right) \in K$. That is,

$$
\delta\left(h_{i}\left(x_{i}\right)\right) \in K, \quad \Delta\left(h_{i}\left(x_{i}\right)\right) \in K .
$$

Let $s_{i}=\operatorname{deg}\left(h_{i}\right)$ and let $L$ be the ideal of $A$ generated by

$$
h_{1}\left(x_{1}\right), h_{2}\left(x_{2}\right), \ldots, h_{n}\left(x_{n}\right) .
$$

For any standard monomial $X=x_{1}^{r_{1}} x_{2}^{r_{2}} \cdots x_{n}^{r_{n}}$, there exist polynomials $q_{1}, \ldots$, $q_{n}, t_{1}, \ldots, t_{n}$ of $\mathbf{k}[x]$ such that

$$
x_{i}^{r_{i}}=q_{i}\left(x_{i}\right) h_{i}\left(x_{i}\right)+t_{i}\left(x_{i}\right), \quad \operatorname{deg}\left(t_{i}\right)<s_{i}
$$

for $i=1,2, \ldots, n$. Replacing each factor $x_{i}^{r_{i}}$ in $X$ by the right hand of the equation (2), we have the fact that $X$ is congruent to a k-linear combination of finite standard monomials

$$
x_{1}^{p_{1}} x_{2}^{p_{2}} \cdots x_{n}^{p_{n}}, \quad p_{i}<s_{i} \text { for } i=1,2, \ldots, n
$$

modulo $L$. Thus $A / L$ is finite dimensional and hence $L$ is a cofinite ideal. 
Note that every element of $L$ is a sum of elements of the form $a h_{i}\left(x_{i}\right) b$, where $a, b \in A$ and $i=1, \ldots, n$. For every element $a h_{i}\left(x_{i}\right) b$, we have

$$
\begin{aligned}
\delta\left(a h_{i}\left(x_{i}\right) b\right)=\delta(a) \Delta\left(h_{i}\left(x_{i}\right)\right) \Delta(b) & +\Delta(a) \delta\left(h_{i}\left(x_{i}\right)\right) \Delta(b) \\
& +\Delta(a) \Delta\left(h_{i}\left(x_{i}\right)\right) \delta(b) \in K .
\end{aligned}
$$

Hence $\{f, g\}(L)=\langle\delta(L), f \otimes g\rangle=0$ and thus $\{f, g\} \in A^{\circ}$.

Step 2. For every $f, g \in A^{\circ},\{f, g\}=-\{g, f\}$ : Since $\tau \circ \delta=-\delta$, we have immediately that

$$
\begin{aligned}
\{f, g\}(x) & =\langle\delta(x), f \otimes g\rangle=\langle\tau \circ \delta(x), g \otimes f\rangle \\
& =-\langle\delta(x), g \otimes f\rangle=-\{g, f\}(x)
\end{aligned}
$$

for all $x \in A$. Thus we have $\{f, g\}=-\{g, f\}$.

Step 3. The equation (1) satisfies the Leibniz rule: Since

$$
\{f g, h\}(x)=\langle(\Delta \otimes 1) \circ \delta(x), f \otimes g \otimes h\rangle
$$

and

$$
\begin{aligned}
& (f\{g, h\}+\{f, h\} g)(x) \\
= & \langle(1 \otimes \delta) \circ \Delta(x), f \otimes g \otimes h\rangle+\left\langle\tau_{23} \circ(\delta \otimes 1) \circ \Delta(x), f \otimes g \otimes h\right\rangle
\end{aligned}
$$

for $x \in A$ and $f, g, h \in A^{\circ}$, it is enough to show that

$$
(\Delta \otimes 1) \circ \delta=(1 \otimes \delta) \circ \Delta+\tau_{23} \circ(\delta \otimes 1) \circ \Delta .
$$

But the equation (3) is just the co-Leibniz rule of $\delta$.

Step 4. The equation (1) satisfies the Jacobi identity: Observe that

$$
\begin{aligned}
& \{\{f, g\}, h\}(x)=\langle(\delta \otimes 1) \circ \delta(x), f \otimes g \otimes h\rangle, \\
& \{\{g, h\}, f\}(x)=\left\langle\tau_{12} \circ \tau_{23} \circ(\delta \otimes 1) \circ \delta(x), f \otimes g \otimes h\right\rangle, \\
& \{\{h, f\}, g\}(x)=\left\langle\tau_{23} \circ \tau_{12} \circ(\delta \otimes 1) \circ \delta(x), f \otimes g \otimes h\right\rangle
\end{aligned}
$$

for $x \in A$ and $f, g, h \in A^{\circ}$. Hence (1) satisfies the Jacobi identity if and only if $\delta$ satisfies

$$
(\delta \otimes 1) \circ \delta+\tau_{12} \circ \tau_{23} \circ(\delta \otimes 1) \circ \delta+\tau_{23} \circ \tau_{12} \circ(\delta \otimes 1) \circ \delta=0 .
$$

But the equation (4) is the co-Jacobi identity of $\delta$. Hence (1) satisfies the Jacobi identity.

Step 5. $\Delta(\{f, g\})=\{\Delta(f), \Delta(g)\}$ for all $f, g, \in A^{\circ}$ : For any $x, y \in A$,

$$
\begin{aligned}
\Delta(\{f, g\})(x \otimes y)= & \{f, g\}(x y)=\langle\delta(x y), f \otimes g\rangle \\
= & \langle\delta(x) \Delta(y), f \otimes g\rangle+\langle\Delta(x) \delta(y), f \otimes g\rangle \\
= & \sum\left\langle\delta(x), f^{\prime} \otimes g^{\prime}\right\rangle\left\langle\Delta(y), f^{\prime \prime} \otimes g^{\prime \prime}\right\rangle \\
& +\sum\left\langle\Delta(x), f^{\prime} \otimes g^{\prime}\right\rangle\left\langle\delta(y), f^{\prime \prime} \otimes g^{\prime \prime}\right\rangle \\
= & \left\{f^{\prime}, g^{\prime}\right\}(x)\left(f^{\prime \prime} g^{\prime \prime}\right)(y)+\left(f^{\prime} g^{\prime}\right)(x)\left(\left\{f^{\prime \prime}, g^{\prime \prime}\right\}\right)(y) \\
= & \{\Delta(f), \Delta(g)\}(x \otimes y),
\end{aligned}
$$


where $\Delta(f)=\sum f^{\prime} \otimes f^{\prime \prime}, \Delta(g)=g^{\prime} \otimes g^{\prime \prime}$. Thus we have

$$
\Delta(\{f, g\})=\{\Delta(f), \Delta(g)\}
$$

for $f, g \in A^{\circ}$. This completes the proof of Theorem 3 .

Refer to $[1,1.3]$ for the definition of Lie bialgebra. Let $(\mathfrak{g}, \delta)$ be a Lie bialgebra, $U(\mathfrak{g})$ the universal enveloping algebra of $\mathfrak{g}$ and $\Delta$ the comultiplication of $U(\mathfrak{g})$. The cobracket $\delta$ is extended uniquely to a $\Delta$-derivation $\bar{\delta}$. That is,

$$
\bar{\delta}: U(\mathfrak{g}) \longrightarrow U(\mathfrak{g}) \otimes U(\mathfrak{g})
$$

is a $\mathbf{k}$-linear map such that $\left.\bar{\delta}\right|_{\mathfrak{g}}=\delta$ and $\bar{\delta}(x y)=\bar{\delta}(x) \Delta(y)+\Delta(x) \bar{\delta}(y)$ for all $x, y \in U(\mathfrak{g})$. Then, by [1, Proposition 6.2.3], $U(\mathfrak{g})$ is a co-Poisson Hopf algebra with Poisson co-bracket $\bar{\delta}$.

Corollary 4. Let $(\mathfrak{g}, \delta)$ be a finite dimensional Lie bialgebra. Then the Hopf dual $U(\mathfrak{g})^{\circ}$ of the universal enveloping algebra $U(\mathfrak{g})$ is a Poisson Hopf algebra with Poisson bracket

$$
\{f, g\}(x)=\langle\bar{\delta}(x), f \otimes g\rangle, \quad x \in U(\mathfrak{g})
$$

for $f, g \in U(\mathfrak{g})^{\circ}$.

Proof. Let $\left\{x_{1}, \ldots, x_{n}\right\}$ be a basis of $\mathfrak{g}$. Then $U(\mathfrak{g})$ is an almost normalizing extension over $\mathbf{k}$ with generators $x_{1}, \ldots, x_{n}$. Thus the result follows immediately from Theorem 3.

\section{References}

[1] V. Chari and A. Pressley, A Guide to Quantum Groups, Cambridge University Press, Providence, 1994.

[2] T. J. Hodges, T. Levasseur, and M. Toro, Algebraic structure of multi-parameter quantum groups, Advances in Math. 126 (1997), 52-92.

[3] A. Joseph, Quantum Groups and Their Primitive Ideals, A series of modern surveys in mathematics, vol. 3. Folge-Band 29, Springer-Verlag, 1995.

[4] J. C. McConnell and J. C. Robson, Noncommutative Noetherian Rings, Pure \& Applied Mathematics, A Wiley-interscience series of texts, monographs \& tracts, Wiley Interscience, New York, 1987.

SeI-Qwon OH

Department of Mathematics

Chungnam National University

DAEJEON 305-764, KOREA

E-mail address: sqoh@cnu.ac.kr

HYUnG-Min PARK

Department of Mathematics

Chungnam National University

DAEJEON 305-764, Korea

E-mail address: my-lovemin@hanmail.net 